\title{
Low-Frequency Characterization of Switched dc-dc Converters
}

G.W. WESTER, Member IEEE

R.D. MIDDLEBROOK, Senior Member, IEEE

California Institute of Technology

Pasadena, Calif. 91109

\begin{abstract}
Averaging techniques are developed to represent buck, boost, and buck-boost types of switched dc-dc converters by approximate continuous models. Simple analytical expressions in terms of the circuit components are derived for the characteristic transient and frequency responses of time-averaged (continuous) power-stage models for use in designing and understanding the behavior of corresponding switched power stages. Novel conclusions include the dependence of effective circuit component values upon switch duty ratio and the existence of a real positive zero in certain transfer functions. Responses from analog computer simulations of the switched and averaged power stages agree well and, in turn, confirm the analytic predictions. High-order systems can be analyzed by the averaging technique without a commensurate increase in complexity.
\end{abstract}

\section{Introduction}

Switched dc-dc converters are composed of two functional blocks, as shown in Fig. 1. The power stage chops, rectifies, and filters an analog signal derived from the source $v_{s}$ to produce an analog output voltage $v$, and the switch controller provides, from an analog signal $\epsilon$, the digital control $d$ necessary to drive the "chopping" switch in the power stage. Fig. 2 shows representative power-stage types (buck, boost, and buck-boost) commonly used in switched converters; the assumed load is resistive $R$, and resistances $R_{l}$ and $R_{c}$ are included to account for parasitic losses exposed by large currents in the physical inductor and capacitor. The nature of circuit operation has been adequately discussed elsewhere [1], and will not be reiterated here. The duty ratio $D$, defined as the fraction of time that the chopping switch is closed, is a control mechanism for varying the dc output voltage. One can assume without loss of generality that the switch is driven by the digital signal $d$ according to

$$
d(t)= \begin{cases}1, & \text { switch closed } \\ 0, & \text { switch open. }\end{cases}
$$

Consequently, $D$ is numerically equal to the dc average of $d(t)$.

When the converter is part of a regulator in which the controller input $\epsilon$ is generated from an appropriate feedback signal, then closed-loop stability becomes important. Stability can be examined if one knows how a disturbance in $\epsilon$ propagates through the controller and power stage to affect $d$ and $v$. A given switch controller can be characterized, at least approximately, by describing-function analysis, but the power stage, because it is a switched nonlinearity not amenable to conventional analysis, has succumbed only to a static description [2] of the dc output in terms of duty ratio. In review, the static ratio of dc output to dc source input varies with duty ratio and is always less than unity for buck, always greater than unity for boost, and either greater or less than unity for buck-boost power stages.

The present objective is to extend the static description of power stages by analyzing dynamic (e.g., transient and

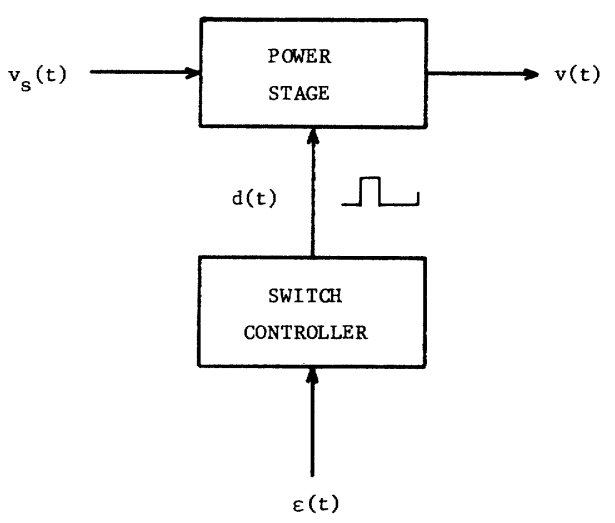

Fig. 1. Block diagram of a power stage and controller.
Presented at the Third IEEE Power Processing and
pecialists Conference, Atlantic City, N.J., May 1972.

Manuscript received May 15, 1972.
IEEE TRANSACTIONS ON AEROSPACE AND ELECTRONIC SYSTEMS

VOL. AES-9, NO. 3 MAY 1973 

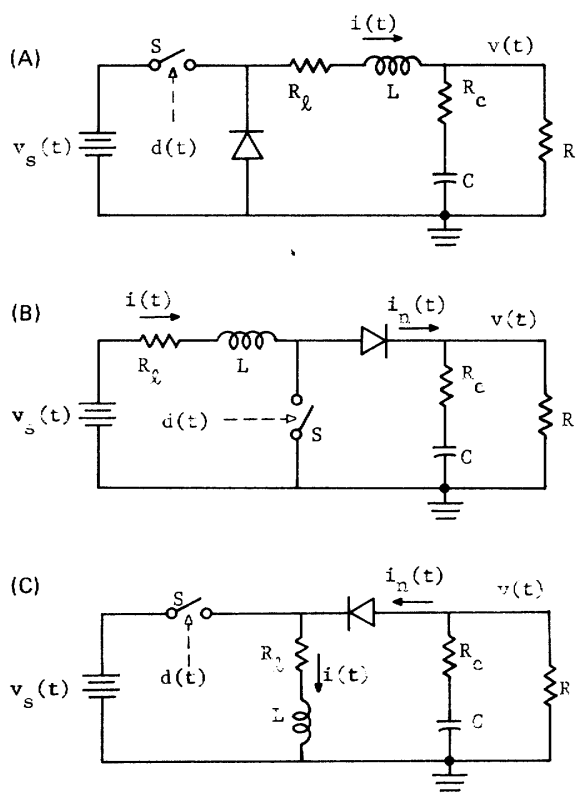

Fig. 2. Circuit configurations of switched power stages: (A) buck, (B) boost, and (C) buck-boost.

sinusoidal) variations of the two power-stage inputs. In essence, this means finding the effective transfer functions which relate $v_{s}$ and $\epsilon$ to power-stage output $v$, even though the power stage is switched and nonlinear. Previous attempts at dynamic analysis were either prematurely stalled [3] before reaching simple equivalent circuits and tractable expressions, or thwarted by poor experimental correlation [4] The objective is attained here by the development of continuous linearized models for the switched power stages in Fig. 2; thus, the power stage and controller can be treated as separate linear blocks.

\section{Averaged Power-Stage Models}

\section{General Models}

Consider first the boost power stage. One can easily verify that Fig. 3 is an exact equivalent circuit $^{1}$ of the boost power stage shown in Fig. 2(B). The factor $1-d(t)$ can be identified as a discontinuous dependent generator gain, and is the principal cause of analytic difficulty. If one is willing to neglect detail in order to study long-range trends, then the forcing functions (sources) may be averaged over a time interval small with respect to the response times of the state variables without appreciably altering the essential nature of circuit response. This concept is the basis of subsequent simplification. Its usefulness arises from the fact that, by design, the state-variable response times are always much greater than the nominal period $T$ of the switch

\footnotetext{
${ }^{1}$ Observe the following notational convention: circles are used to denote independent sources, whereas squares represent dependent generators.
}

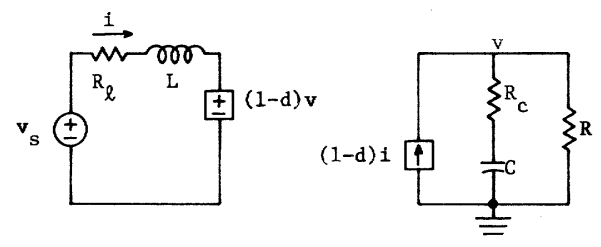

Fig. 3. Equivalent circuit of switched boost power stage.

Fig. 4. Averaged power-stage models: (A) boost, (B) buck-boost, (C) buck, and (D) simplified buck.
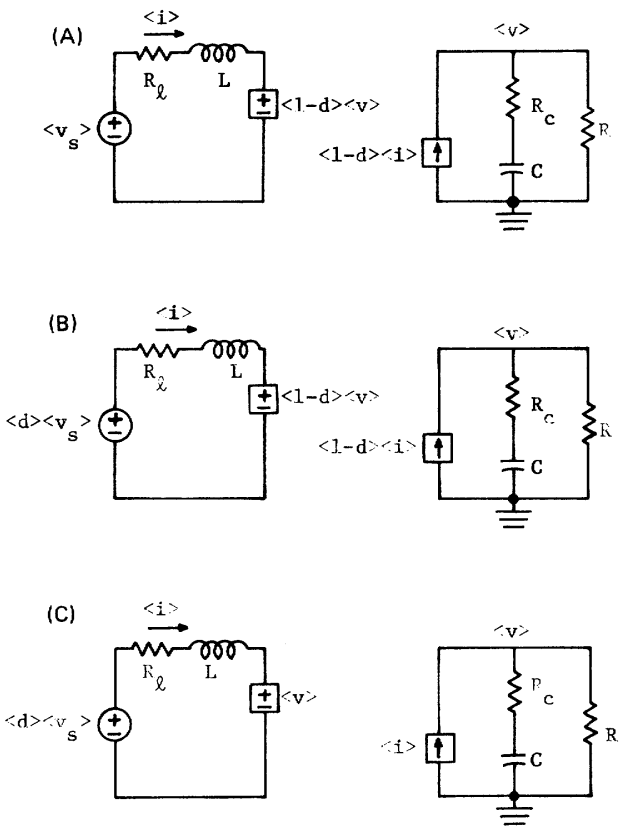

(D)

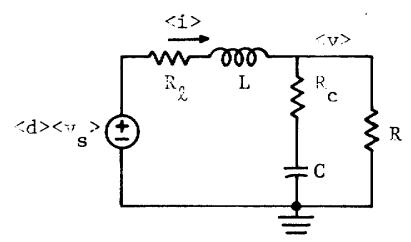

controller, and, therefore, the averaging interval can be comparable to the switching period in order to average the factor $1-d$. A possible definition of the averaging operation, which is useful for the extraction of low-frequency components from $d$, is given [1] by

$$
<d>(t)=\frac{1}{T} \int_{t-T}^{t} d(x) d x
$$

The effect of averaging is approximately that of a low-pass filter with cut-off frequency $\omega_{s}=2 \pi / T$.

The time-averaged model of the boost power stage is shown in Fig. 4(A). The model is useful only for frequenices less than $\omega_{s}$, since the switching ripple has been averaged 


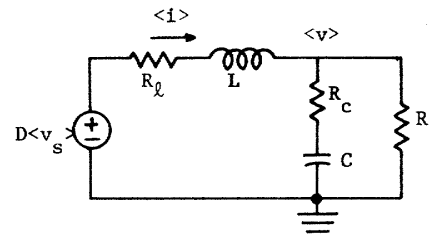

Fig. 5. Equivalent circuit of the averaged buck power-stage model for source variations and constant control.

out. Although the model is still basically nonlinear, the dependent generator gains are now continuous. In the same manner, averaged models of the buck-boost and buck power stages, respectively, are obtained and illustrated in Fig. 4(B) and (C). Because its dependent generator gains are unity, the averaged buck power stage can be simplified to the linear equivalent circuit in Fig. 4(D).

The basic models have now been formulated to analyze the slowly varying average envelope of power-stage responses. Transient and frequency analysis of the response to the two power-stage inputs, source and control, will be investigated next; however, since superposition does not apply for nonlinear circuits, a particular response is meaningful only if both inputs are specified.

\section{Analysis of Response to Source Variations}

Assume for simplicity that the averaged control is constant $t^{2}$ :

$$
<d>(t)=D .
$$

The equivalent circuit which results when Fig. 4(D) is specialized by the above substitution is shown in Fig. 5, and relates unspecified source variations to the corresponding output variations for the buck power stage. In applying the same procedure to the boost power stage, one can define a complementary duty ratio $D^{\prime}$ as

$$
D^{\prime} \equiv 1-D,
$$

which, with (3) and Fig. 4(A), yields the averaged model shown in Fig. 6(A). The dependent generators can be eliminated by normalizing the constant generator gains to unity in the following manner. If in the inductor loop one divides the voltage sources and impedance values by the factor $D^{\prime}$, the current $\langle i\rangle$ remains unchanged. After the current generator gain has been similarly normalized to unity, the equivalent circuit shown in Fig. 6(B) can be further simplified to the linear circuit model in Fig. 6(C). Development of the corresponding buck-boost model is entirely analogous to that of the boost model, so only the final

\footnotetext{
${ }^{2}$ As previously noted, that constant is numerically equal to the duty ratio.
}
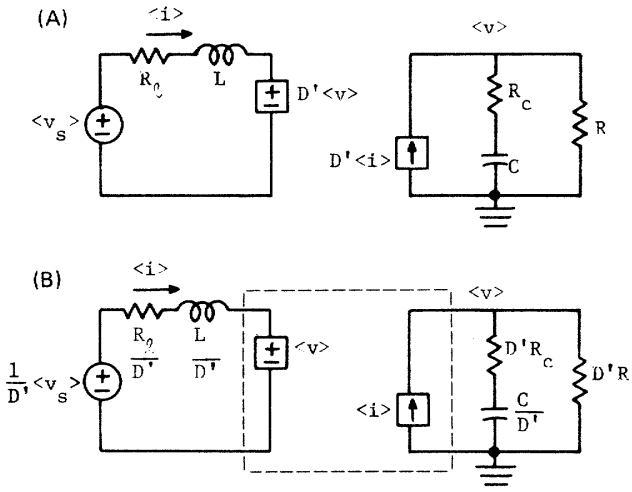

(C)

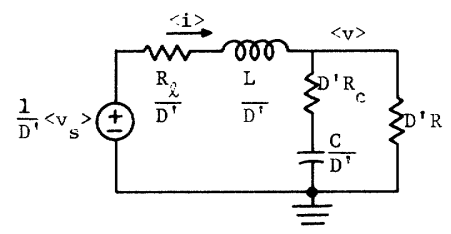

Fig. 6. Reduction of the averaged boost powerstage model for source variations and constant control.

Fig. 7. Equivalent circuit of the averaged buck-boost power-stage model for source variations and constant control.

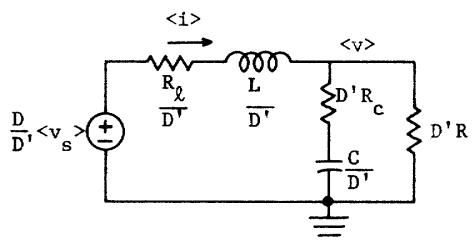

simplified circuit, which results from the assumption in (3), is shown in Fig. 7.

The averaged circuits in Figs. 5, 6(C), and 7 are linear and, hence, useful for analysis of either transient or frequency responses caused by variations in the source voltage. When the source is held constant, they also apply for transient analysis caused by the control input, since the control is constant following a step change. Although the circuit topology for each power-stage type is identical, notice how the effective circuit component values for the boost and buck-boost power stages are modified by the (complementary) duty ratio. When Laplace transform theory is applied to each type of power stage to find the source-input transfer function $G_{s}(s)$, defined as

$$
\left.G_{s}(s) \equiv \frac{V(s)}{V_{s}(s)}\right|_{\text {zero initial conditions }}
$$

where $V(s)$ and $V_{s}(s)$ are the Laplace transforms of $v(t)$ and $v_{s}(t)$, respectively, the result can be expressed as

$$
G_{s}(s)=A_{s 0} G_{f}(s)
$$


Summary of Analytic Parameters in Power-Stage Transfer Functions

\begin{tabular}{|c|c|c|c|c|}
\hline & Buck & Boost & Buck-Boost & \\
\hline$A_{\text {so }}$ & $\mathrm{D}$ & $1 / D^{\prime}$ & $\mathrm{D} / \mathrm{D}^{\prime}$ & \\
\hline $\mathrm{A}_{\mathrm{CO}}$ & $\mathrm{V}_{\mathrm{s}}$ & $\frac{\left(D^{\prime}\right)^{2} R-R_{\ell}}{\left(D^{\prime}\right)^{2} R+R_{\ell}} \frac{V_{s}}{\left(D^{\prime}\right)^{2}}$ & $\frac{\left(D^{\prime}\right)^{2} R-\left(D-D^{\prime}\right) R_{\ell}}{\left(D^{\prime}\right)^{2} R+R_{\ell}}$ & $\frac{V_{s}}{\left(D^{\prime}\right)^{2}}$ \\
\hline$G_{\text {fo }}$ & $\frac{R}{R+R_{\ell}}$ & $\frac{R}{R+R_{\ell} /\left(D^{\prime}\right)^{2}}$ & $\frac{R}{R+R_{\ell} /\left(D^{\prime}\right)^{2}}$ & \\
\hline$\omega_{0}$ & $\frac{1}{\sqrt{L C}} \sqrt{\frac{R+R_{\ell}}{R+R_{C}}}$ & $\frac{1}{\sqrt{L C}} \sqrt{\frac{\left(D^{\prime}\right)^{2} R+R_{\ell}}{R+R_{c}}}$ & $\frac{1}{\sqrt{L C}} \sqrt{\frac{\left(D^{\prime}\right)^{2} R+R_{\ell}}{R+R_{C}}}$ & \\
\hline Q & $\frac{1}{\omega_{o}}\left[\mathrm{CR}_{\mathrm{C}}+\frac{\mathrm{CRR}_{\ell}+\mathrm{L}}{\mathrm{R}+\mathrm{R}_{\ell}}\right]^{-1}$ & $\frac{1}{\omega_{0}}\left[\mathrm{CR}_{C}+\frac{\mathrm{CRR}_{\ell}+\mathrm{L}}{\left(D^{\prime}\right)^{2} \mathrm{R}+\mathrm{R}_{\ell}}\right]^{-1}$ & $\frac{1}{\omega_{0}}\left[\mathrm{CR}_{c}+\frac{\mathrm{CRR}_{\ell^{+}} \mathrm{L}}{\left(\mathrm{D}^{\prime}\right)^{2} \mathrm{R}+\mathrm{R}_{\ell}}\right.$ & -1 \\
\hline$w_{z}$ & $\frac{1}{\mathrm{CR}}$ & $\frac{1}{\mathrm{CR}_{\mathrm{c}}}$ & $\frac{1}{\mathrm{CR}_{\mathrm{C}}}$ & \\
\hline$w_{a}$ & $\infty$ & $\frac{\left(D^{\prime}\right)^{2} R-R_{\ell}}{L}$ & $\frac{\left(D^{\prime}\right)^{2} R-\left(D-D^{\prime}\right) R_{\ell}}{D L}$ & \\
\hline
\end{tabular}

Fig. 8. Block diagram of the averaged powerstage model for source variations and constant control.

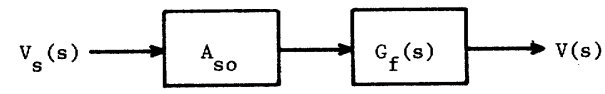

where

$$
G_{f}(s)=G_{f 0} \frac{1+\frac{s}{\omega_{z}}}{1+\frac{1}{Q} \frac{s}{\omega_{0}}+\left(\frac{s}{\omega_{0}}\right)^{2}} .
$$

Equation (6) can be interpreted as the transfer function of an amplifier in series with a filter, as shown in Fig. 8. Analytic expressions for the normalized filter and amplifier factors are listed in Table I for each type of power stage. Observe the effect of duty ratio on selected corner frequencies and on the quality factor of the filter as a consequence of modified effective component values.

\section{Analysis of Response to Control Variations}

Consider now the situation when the averaged source voltage is a constant $V_{s}$,

$$
\left\langle v_{s}\right\rangle(t)=V_{s},
$$

and the averaged control is fluctuating with time. The buck power stage is readily investigated by using the above substitution in Fig. 4(D) to obtain Fig. 9, which is a linear circuit with constant component values. However, the averaged
Fig. 9. Equivalent circuit of the averaged buck power-stage model for control variations and a constant source.

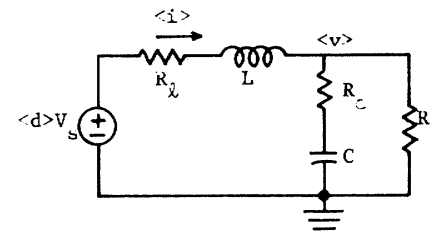

boost and buck-boost models are nonlinear for variations of the control, so a different approach is required.

The transient responses of the various power stages caused by step changes in averaged control are directly available from the equivalent circuits in the preceding subsection when the averaged source is constant, so in the present subsection, the effect of an arbitrary control perturbation on the output is sought. Assume the averaged control is given by

$$
<d>(t)=D+\hat{d}(t)
$$

where $\hat{d}$ is a time-varying perturbation of the duty ratio $D$. Based on (4) and (9), one can show that

$$
<1-d>(t)=D^{\prime}-\hat{d}(t) .
$$

The control perturbation causes corresponding perturbations of the averaged state variables, as expressed by

$$
\begin{aligned}
& \langle v>(t)=V+\hat{v}(t) \\
& \langle i>(t)=I+\hat{i}(t) .
\end{aligned}
$$



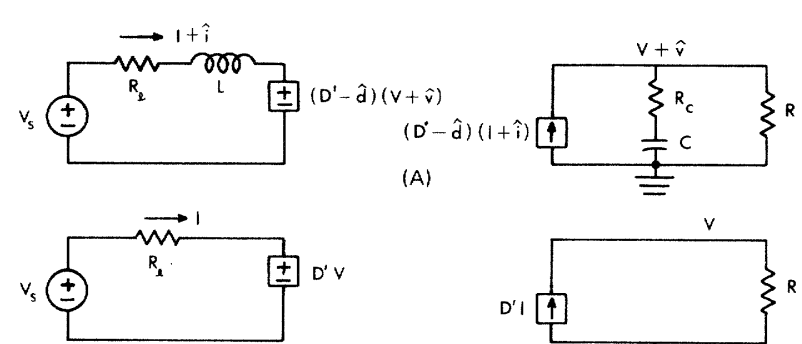

(B)
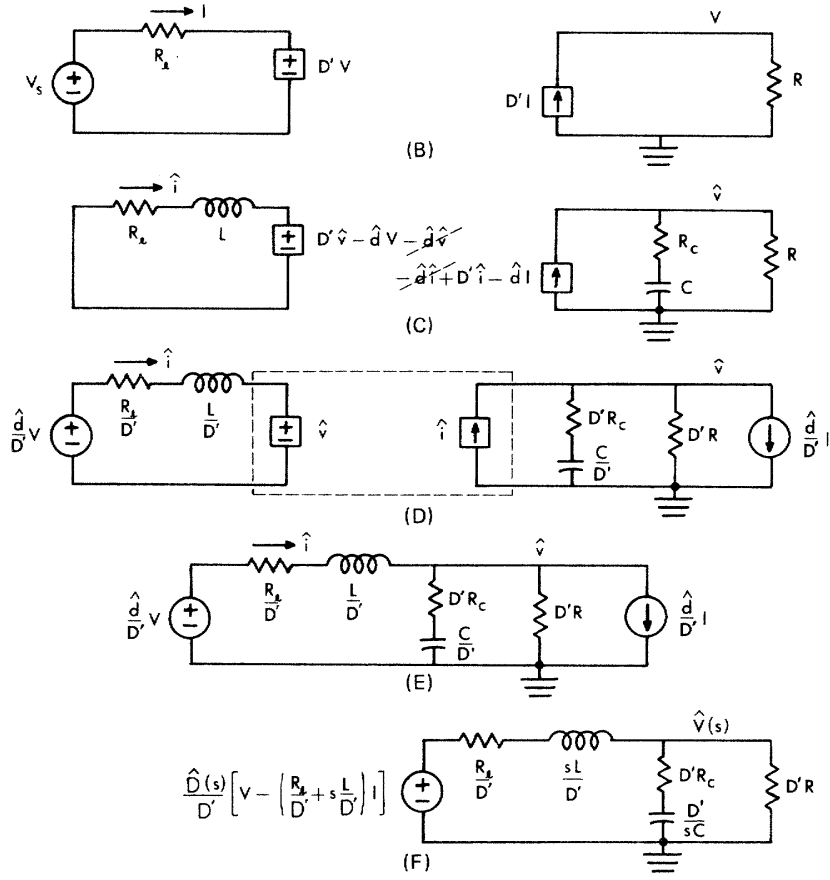

Fig. 10. Reduction of the averaged boost power-stage model for small control variations and a constant source.

The problem is the find $\hat{v}$ in terms of $\hat{d}$. The equivalent circuit of the averaged boost power stage, which results when (8) through (12) are substituted into Fig. 4(A), is shown in Fig. 10(A). After the unperturbed values of the state variables are evaluated from the steady-state equivalent circuit in Fig. 10(B) and subtracted from Fig. 10(A), the equivalent circuit which remains for perturbations is shown in Fig. 1O(C). The circuit in Fig. 10(C) can be linearized by restricting the perturbation amplitude in order to make the second-order terms $\hat{d} \hat{v}$ and $\hat{d} \hat{i}$ negligibly small with respect to the other generator terms:

$$
\hat{v} \ll<, \quad \hat{i}<<I \quad \text { for small-amplitude } \hat{d} \text {. }
$$

For each generator, one of the remaining terms is proportional to the independently forced control perturbation, while the other is proportional to a circuit-dependent perturbation, so that meaningful separations into dependent and independent generators can be accomplished. Following the procedure described in the preceding subsection, one can normalize to unity the gains of the dependent generators to reveal the circuit illustrated in Fig. 10(D). The dotted section of Fig. 10(D) should be recognized as an ideal unitygain transformer, so it can be simplified as shown in Fig. 10(E). The presence of two generators in Fig. 10(E) obscures the relationship between $\hat{d}$ and $\hat{v}$, but since the circuit is linear, Laplace transforms can be manipulated using Thevenin and Norton equivalents to combine the generators
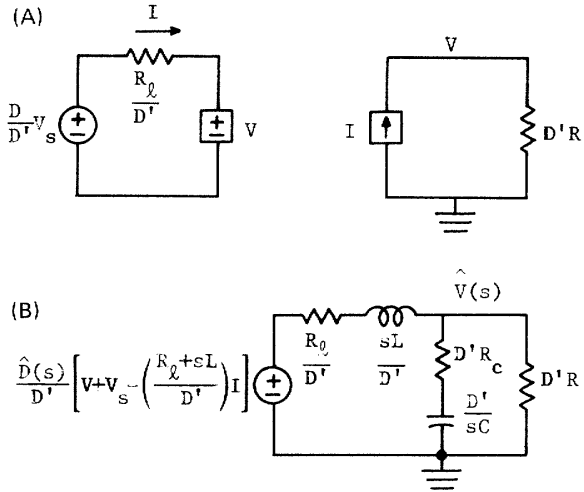

Fig. 11. Equivalent circuits of the averaged buck-boost power-stage model for small control variations and a constant source. (A) Steady state. (B) Linearized for variations.

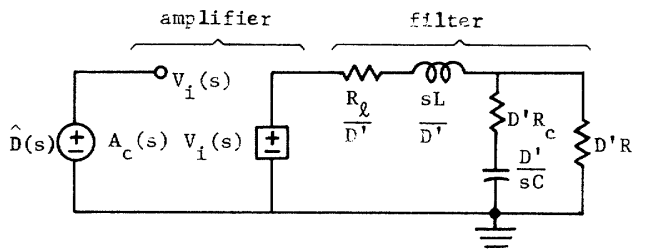

Fig. 12. Equivalent circuit showing an effective amplifier in the averaged boost and buck-boost power-stage models for small control variations and a constant source.

into the single source shown in Fig. 10(F). The equivalent circuit is now in the Laplace transform domain, where $\hat{D}(s)$ and $\hat{V}(s)$ are the Laplace transforms of $\hat{d}(t)$ and $\hat{v}(t)$, respectively.

The procedure just outlined for the boost power stage is also applicable to the buck-boost power stage. The steadystate and perturbation components of the output voltage are easily derived for the buck-boost power stage from Fig. 11 (A) and (B), respectively. The equivalent circuit for perturbations was linearized by (13), as before.

Linearized equivalent circuits for small-amplitude control perturbations have been derived for each power stage. The factors which multiply $\hat{D}(s)$ in the equivalent voltage generators in Figs. 10(F) and 11(B) can be identified as the transfer function of an effective amplifier $A_{c}(s)$, as shown in Fig. 12. Thus, the linearized control-input transfer function $G_{c}(s)$,

$$
\left.G_{c}(s) \equiv \frac{\hat{V}(s)}{\hat{D}(s)}\right|_{\text {zero initial conditions, }}
$$

for each power-stage type can be written in the form

$$
G_{c}(s)=A_{c}(s) G_{f}(s)
$$

where

$$
A_{c}(s)=A_{c 0}\left(1-\frac{s}{w_{a}}\right)
$$




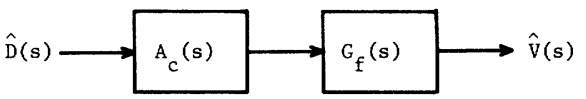

Fig. 13. Linearized block diagram of the averaged power-stage model for small control variations and a constant source.

and $G_{f}(s)$ is the filter transform function given by (7). Analytic expressions for the normalized filter and amplifier factors are contained in Table I. The duty ratio has the same influence on effective component values as observed for source variations. Notice, in addition, the unusual form of the effective amplifier transfer function for boost and buck-boost power stages; the real zero is positive for $D^{\prime}>D_{0}^{\prime}$, where

$$
D_{0}^{\prime}=\left\{\begin{array}{l}
\sqrt{\frac{R_{l}}{R}}, \text { buck } \\
\sqrt{\left(1+\frac{R_{l}}{R}\right) \frac{R_{l}}{R}}-\sqrt{\frac{R_{l}}{R}}, \text { buck-boost. }
\end{array}\right.
$$

Since $R_{l} / R$, and consequently $D_{0}^{\prime}$, is typically small, $\omega_{a}$ is usually positive, so that both the phase lag and amplitude of $A_{c}(j \omega)$ increase with $\omega$. Fig. 13 shows the block diagram of $G_{c}(s)$, in order to expose the similarity of interpretation of (15) and (6).

Rather unusual analytical results have been derived from the averaged power stages. To the authors' knowledge, no tractable analysis of the transient or frequency response associated with a control variation has appeared in the literature for boost or buck-boost power stages. However, Kossov [2] has performed an exact static analysis of the source-to-output gain for the three basic power stages; so, for comparison, the corresponding gains will be derived from the averaged power-stage models.

For static conditions expressed by (3) and (8), the averaged power-stage models are particularly simple, since there is no capacitor current or inductor voltage in the steady state. The static source-to-output gain of each power-stage configuration is easily derived [1]:

$$
\begin{aligned}
& \frac{V}{V_{s}}=\frac{D R}{R_{l}+R}, \quad \text { buck } \\
& \frac{V}{V_{s}}=\frac{D^{\prime} R}{R_{l}+\left(D^{\prime}\right)^{2} R}, \quad \text { boost } \\
& \frac{V}{V_{s}}=\frac{D D^{\prime} R}{R_{l}+\left(D^{\prime}\right)^{2} R}, \quad \text { buck-boost. }
\end{aligned}
$$

It may be observed that, with corresponding notation, (18) through (20) agree precisely with Kossov's equations, (6a), $(6 b)$, and (6c).

Though not analytically founded, a hypothesis postulated by Wells et al. [5] states that the lowest corner frequency in the open-loop boost control-input describing function varies proportionately with $D^{\prime}$. Wells' hypothesis was reportedly supported by experimental observations of a particular boost configuration with additional input and output filtering. The averaged model of the boost power stage under consideration here has a quadratic pole with break frequency $\omega_{0}$, given in Table I, which does, in fact, vary approximately with $D^{\prime}$ for typical circuit values and operating conditions:

$$
\omega_{0} \simeq \frac{D^{\prime}}{\sqrt{L C}} \sqrt{\frac{R}{R+R_{c}}} \text { for } D^{\prime}>>D_{0}^{\prime}, \text { boost. }
$$

To the extent that the effective source impedance (source impedance, input filter, and boosting inductor) is inductive and the effective load impedance (output filter and load) is capacitive, Wells' hypothesis may be a general result. Notice, however, that the corner frequency of the effective amplifier is a stronger function of duty ratio:

$$
\omega_{a} \simeq\left(D^{\prime}\right)^{2} \frac{R}{L}, \text { for } D^{\prime} \gg>D_{0}^{\prime} \text {, boost. }
$$

\section{Open-Loop Behavior}

Of the two input variations considered in the preceding section, responses to control variations are considerably more interesting because the averaged power-stage models are nonlinear with respect to control variations. Analog computer simulations [1] of the switched power stages in Fig. 2 and the corresponding averaged power stages in Fig. 4 are subjected to transients and sinusoidal perturbations of the control for comparison with the analytic expressions just derived; but, first, a specific switch controller is chosen to operate the switched power stage.

\section{Switch Controller}

A pulsewidth modulator (PWM) is used to control the switches in the computer simulation of the power stages in Fig. 2. The PWM samples the controller input $\epsilon$ at uniform time intervals to initiate a sequence of output pulses whose durations are proportional to the sampled input values:

$$
d(t)= \begin{cases}1, & 0 \leqslant t-n T<\tau_{n} \\ 0, & \text { otherwise }\end{cases}
$$

where $n$ is an integer and

$$
\frac{\tau_{n}}{T}= \begin{cases}0, & \epsilon(n T)<0 \\ \epsilon(n T), & 0 \leqslant \epsilon(n T)<1 \\ 1, & 1<\epsilon(n T) .\end{cases}
$$

To compare computer results with analysis, one should use a simple analytic controller model. Step changes of $\epsilon$ 
(A)

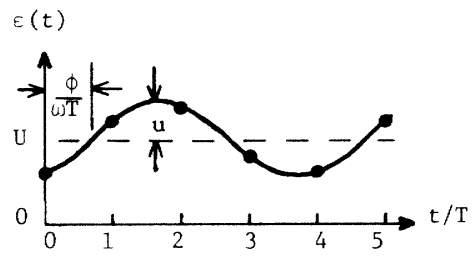

(B)

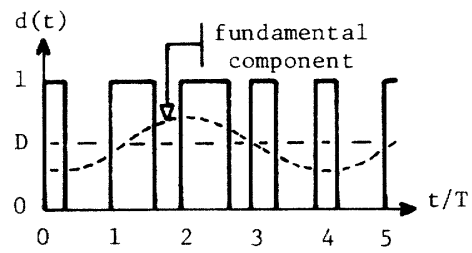

Fig. 14. PWM response to sinusoidal modulation. (A) Input. (B) Output.

manifest themselves in the controller output within a switching period $T$, and the controller output remains unchanged thereafter. Since $T$ is much less than the response time of the power stage, the PWM does not significantly affect the overall transient response. Sinusoidal control perturbations are a different matter, however; if the dimensionless controller input, as shown in Fig. 14(A), is given by

$$
\epsilon(t)=U+u \sin (\omega t-\phi)
$$

where $U+u<1$ and $U-u>0$, then the spectrum of the controller output shown in Fig. 14(B) can be evaluated by the extension [1] of a tedious two-dimensional Fourier analysis [6]:

$$
\begin{gathered}
d(t)=U+\sum_{\substack{m=-\infty \\
m \neq 0}}^{\infty} \frac{1}{j m \omega_{s} T} \exp \left(j m \omega_{s} t\right) \\
-\sum_{m=-\infty}^{\infty} \sum_{n=-\infty}^{\infty} \exp [-j n(\phi+\pi)] \\
\cdot \frac{J_{n}[u \Omega T]}{j \Omega T} \exp [j \Omega(t-U T)] \\
\Omega \equiv m \omega_{s}+n \omega \neq 0
\end{gathered}
$$

where $J_{n}[z]$ is a Bessel function of the first kind.

Whether $\omega$ and $\omega_{s}$ are commensurable or not, the describing function of the PWM can be approximated well for small $u$ by $\exp (-j \omega U T)$. Thus, the frequency response of the PWM can be modeled by a phase lag which increases linearly with modulation frequency $\omega$.

\section{Component Values}

The following numerical values are consistent with typical design constraints $\left(L / R_{l} \gg T, R C \gg>T, 2 L / R>T\right)$ and will be used henceforth for specific analysis:

$$
\begin{aligned}
& T=10^{-4} \text { second } \\
& R=60 \text { ohms } \\
& L=6.10^{-3} \text { henry } \\
& C=(1 / 24) 10^{-3} \text { farad }=41.7 \mu f \\
& R_{l}=3 \mathrm{ohms} \\
& R_{c}=1 \mathrm{ohm} \\
& V_{s}=60 \text { volts. }
\end{aligned}
$$

\section{Transient Response}

It is convenient to record on a strip chart the transient response of switched and averaged converter models as simulated on the analog computer. Whereas step transitions of $\epsilon$ and $\langle d\rangle$ between all permutations of the levels $0.25,0.50$, and 0.75 were investigated, only representative transitions for the boost power stage are shown here. Fig. 15 shows corresponding experimental transient responses of the switched and averaged models, for comparison. The excellent correlation is typical of all power stages and control transitions. Notice, qualitatively, how the damping factor and natural frequency depend on duty ratio, as predicted.

\section{Frequency Response}

A simple analytic description of converter frequency response results when the PWM describing function is multiplied with the linearized control-input power-stage describing function $G_{c}(j \omega)$ in (15). A Bode plot of the theoretical frequency response of a boost converter is shown in Fig. 16 for several values of duty ratio.

Experimental frequency response is measured by enforcing a control input in the form of (25). At any given modulation frequency $\omega$, the control input and power-stage output are simultaneously recorded on a strip chart, from which the amplitude and phase of the output component at the modulation frequency can be measured with respect to the modulation amplitude and phase. The amplitude and phase of the effective transfer function are then located on a Bode diagram. Fig. 17 shows experimental data from the switched and averaged models of the boost power stage superimposed on the theoretical frequency response for $D=0.50$. The degree of correlation in evidence is typical of the other power stages and duty ratios investigated.

The scattering of switched data at higher frequencies in Fig. 17 leaves uncertain the role of various theoretical factors in determining the overall frequency response. To study this question, one can decompose the theoretical transfer function into distinct factors which represent the effective amplifier, filter, and switch controller of the aver- 
(A)
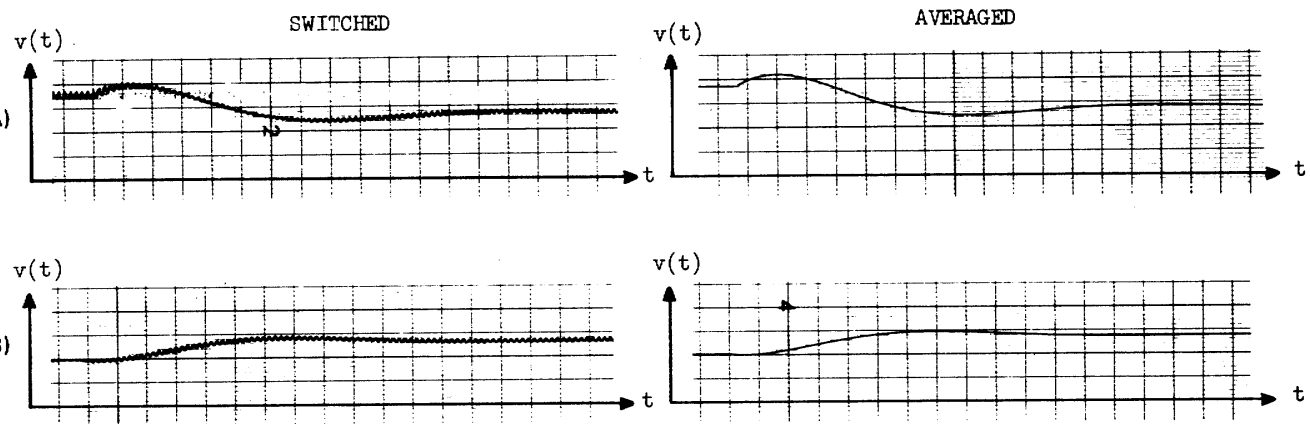

(C)
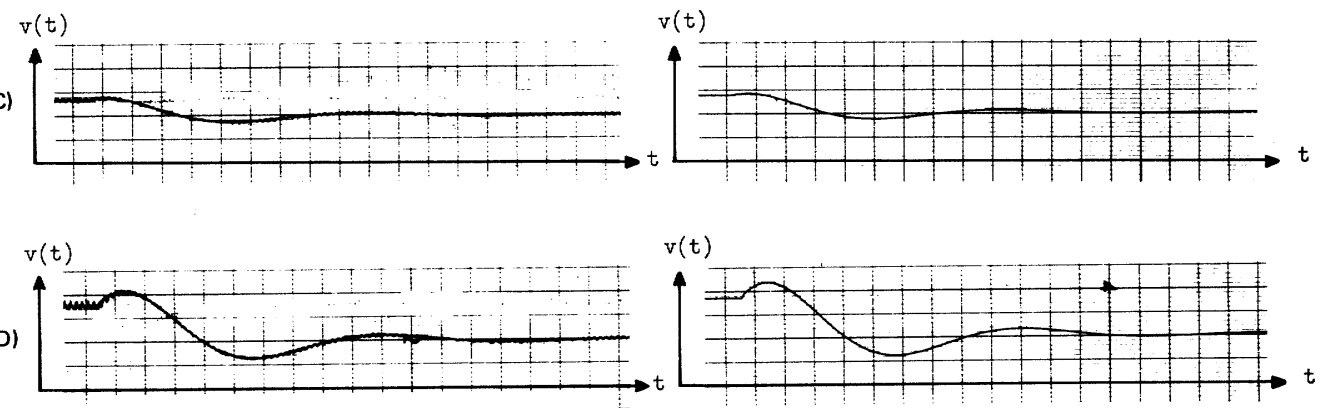

Fig. 15. Experimental transient responses of switched and averaged boost power-stage models. Control transitions are: (A) $0.75 \rightarrow 0.50$, (B) $0.25 \rightarrow 0.50$, (C) $0.50 \rightarrow 0.25$, and (D) $0.75 \rightarrow 0.25$. (Scale factors: 36 $\mathrm{V} / \mathrm{div}$ vertical, $0.5 \mathrm{~ms} / \mathrm{div}$ horizontal.)

Fig. 16. Theoretical control-input frequency response of the boost converter.
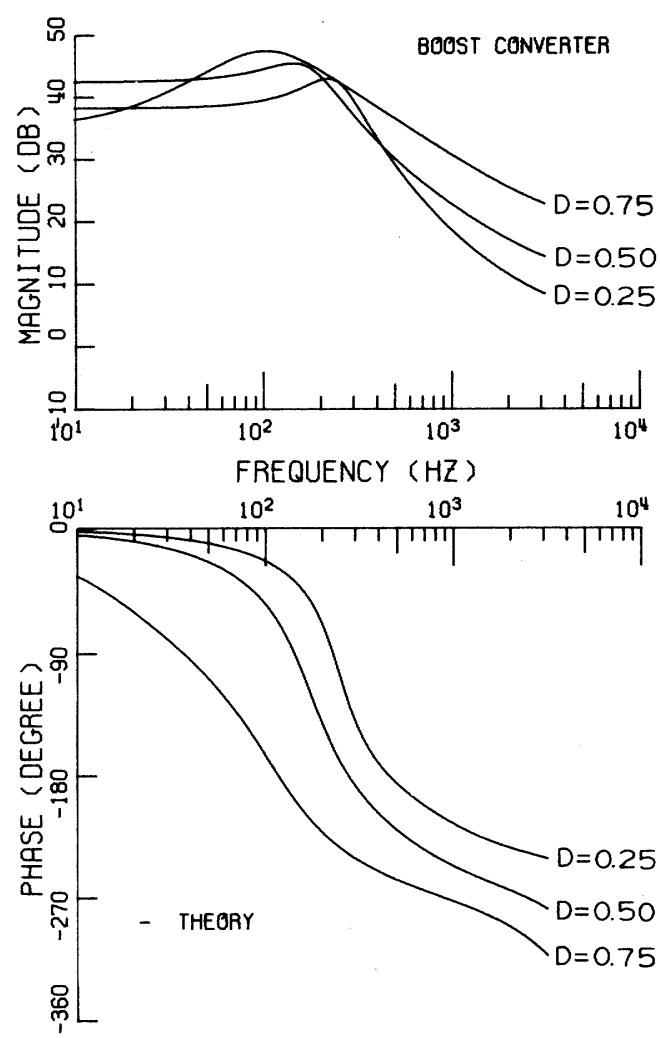

Fig. 17. Experimental control-input frequency response of the boost converter for $D=0.50$.
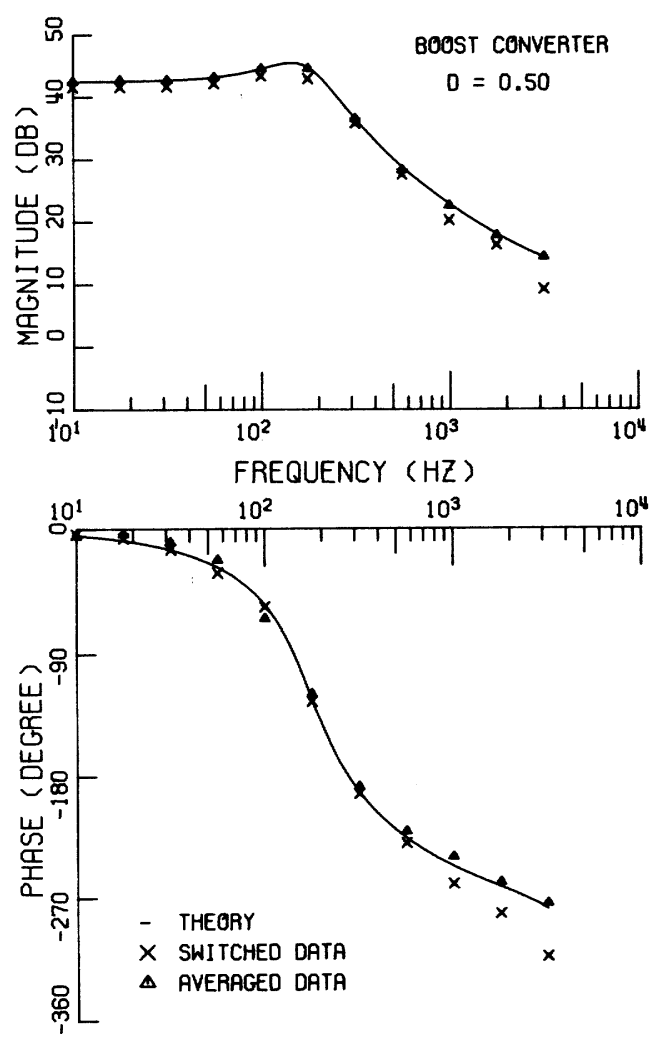


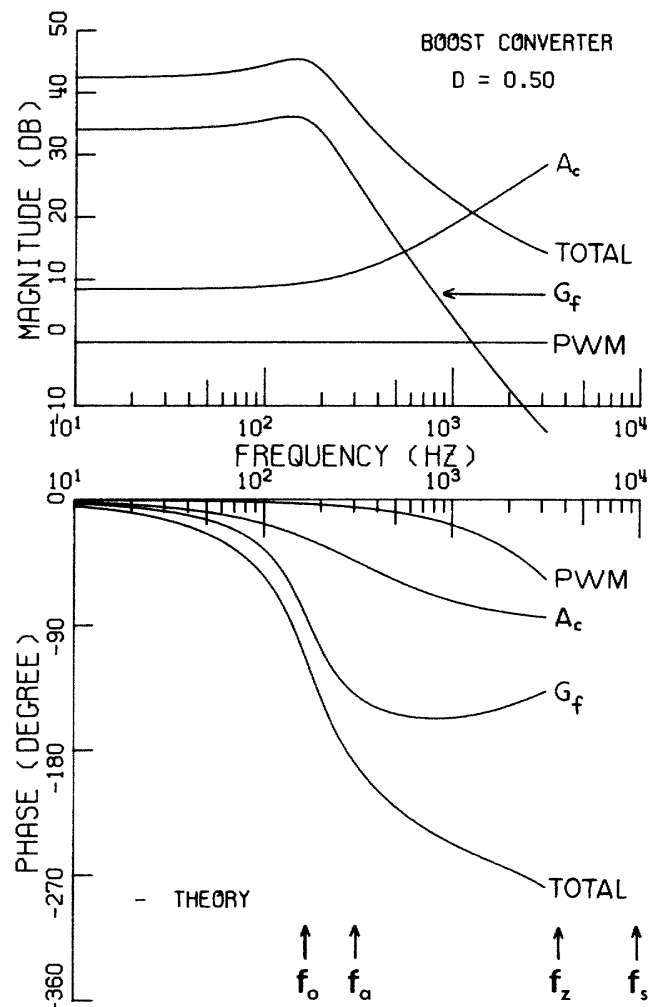

Fig. 18. Components of the theoretical controlinput frequency response of the boost converter for $D=0.50$.

aged boost power stage. These component factors ${ }^{3}$, together with the composite response, are plotted as a function of frequency in Fig. 18, and show that data correlation with the computed curve in Fig. 17 would be much worse if any single theoretical factor were missing. In particular, the presence of the effective amplifier term, novel because of its real positive zero, has been confirmed.

\section{Closed-Loop Behavior}

Given that the open-loop frequency response of switched power stages is approximated by that of the averaged models, one should investigate how well the closed-loop behavior of the switched system can be predicted by the averaged system. One must remember that validity of stability predictions from the averaged model is inherently limited to frequencies less than the switching frequency. The objective of this section is to provide a comparison between theoretical closed-loop stability of averaged systems and experimental stability measurements of switched systems.

The feedback configuration used for stability analysis is shown in Fig. 19, and was designed to make the dc controller output independent of the feedback factor $K$. When $K=0$

\footnotetext{
${ }^{3}$ For convenient amplitude normalization, the scale factor $V_{S}$ is divided from the amplifier factor and multiplied with the filter factor.
}

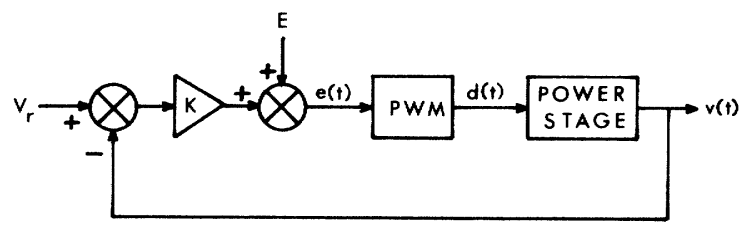

Fig. 19. Converter in a closed-loop regulator configuration.

(no feedback), the dc controller input $U$ sets values for the static PWM output $D$ and the static output voltage $V$. If $V_{r}$ is chosen equal to $V$, then the static output voltage remains constant as $K$ increases.

Local stability can be experimentally examined by gradually increasing the value of the gain factor $K$ until a small disturbance in the steady-state limit cycle no longer decays with time, but grows in amplitude. The critical value of $K$ which separates the two modes of behavior is denoted $K_{c}$, and the corresponding oscillation occurs at frequency $\omega_{c}$. Theoretical values for $K_{c}$ and $\omega_{c}$ are computed from expressions for the frequency response of the averaged models by observing the frequency $\omega_{c}$ where the phase lag is $\pi^{\prime}$, and then computing the gain factor $K_{c}$ which makes the magnitude of the open-loop gain equal to unity.

Experimental values of $K_{c}$ and $\omega_{c}$ are compared in Table II with predicted analytical values. Experimental values could not be obtained in the buck-boost simulation for $D=0.25$ because, as $K$ increases, the discontinuities in output voltage, which are a consequence of switched current through the parasitic resistance of the imperfect filter capacitor, drive the switch controller into a saturated condition before the system becomes unstable. However, one should conclude from Table II that empirical closed-loop stability data from the switched simulation correlates well, overall, with values derived analytically from the averaged models.

\section{Conclusions}

A technique to characterize the low-frequency response of switched power stages has been developed and applied to the simple analytic evaluation of source- and control-input describing functions. Analysis of continuous models which approximate the behavior of switched converters reveals several interesting characteristics, including, for boost and buck-boost power stages, the modification of effective component values by the switch duty ratio and the typical existence of a real positive zero in the linearized control-input describing function. The pulsewidth modulator as a switch controller exerts only weak influence on the frequency response in comparison with the power stage. The averaging technique can include parasitic effects, such as realistic switch and diode models, in the analysis. A computer simulation demonstrates that both open- and closed-loop responses of switched power stages are predicted well by continuous models. Thus, the averaging technique should be a useful tool for the design and analysis of switched converters. 
Critical Stability Factors of the Closed-Loop Regulator Configuration

\begin{tabular}{l|c|c|c|c|c}
\hline \hline \multirow{2}{*}{} & \multirow{2}{*}{$\mathrm{D}$} & \multicolumn{2}{|c|}{$\mathrm{K}_{\mathrm{c}}$} & \multicolumn{2}{c}{$\omega_{\mathrm{c}}\left[10^{3} \mathrm{rad} / \mathrm{sec}\right]$} \\
\cline { 3 - 6 } & & Theory & Measured & Theory & Measured \\
\hline \multirow{3}{*}{ Boost } & 0.25 & 0.028 & 0.034 & 2.84 & 2.87 \\
& 0.50 & 0.012 & 0.015 & 1.73 & 1.65 \\
& 0.75 & 0.004 & 0.007 & 0.73 & 0.82 \\
\hline \multirow{3}{*}{ Buck- } & 0.25 & 0.158 & - & 6.34 & - \\
Boost & 0.50 & 0.023 & 0.037 & 2.37 & 1.89 \\
& 0.75 & 0.006 & 0.010 & 0.93 & 1.13 \\
\hline
\end{tabular}

\section{References}

[1] G. W. Wester, "Low-frequency characterization of switched dc-dc converters," Ph.D. thesis, California Institute of Technology, Pasadena, May 1972.

[2] O. A. Kossov, "Comparative analysis of chopper voltage regulators with LC filters," IEEE Trans. Magnetics Vol. MAG-4, pp. 712-715, December 1968.

[3] L. R. Poulo and S. Greenblatt, "Research investigations on feedback techniques and methods for automatic control," Bose Corp., Natick, Mass., Contract ECOM-0520-F, pp. 156-165, April 1969.
[4] E. E. Landsman, "Modulor converters for space power systems," Power Conditioning Specialists Conf. Rec. (NASAGoddard SFC, Greenbelt, Md.), pp. 87-99, April 1970.

[5] B. A. Wells, B. T. Brodie, and I. M. H. Bábaá, "Analog computer simulation of a dc-to-dc flyback converter," Suppl. to IEEE Trans. Aerospace and Electronic Systems, Vol. AES-3, pp. 399-409, November 1967.

[6] H. E. Rowe, Signals and Noise in Communications Systems. Princeton, N.J.-Van Nostrand, 1965, pp. 257-280.
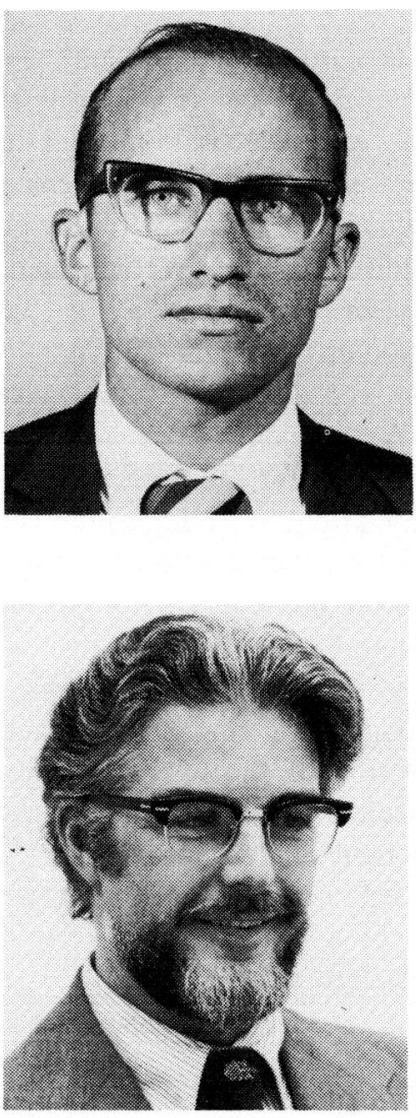

Gene W. Wester (S'68-M'72) was born on March 20, 1944. He received the B.S.E.E. degree from the University of Kansas, Lawrence, in 1967, and the M.S. and Ph.D. degrees in electrical engineering from the California Institute of Technology, Pasadena, in 1968 and 1972 , respectively.

While studying at the Califronia Institute of Technology, he held National Science Foundation Fellowships and Teaching Assistantships. He is presently involved with advanced development of power conditioning at the Jet Propulsion Laboratory, Pasadena.

Dr. Wester is a member of Sigma Xi, Tau Beta Pi, and Eta Kappa Nu.

R. D. Middlebrook (M'56-M'58) was born in England on May 16, 1929. He received the B. A. and M. A. degrees from Cambridge University, England, and the M. S. and Ph.D. degrees in electrical engineering from Stanford University, Stanford, Calif. He was an Electronics Instructor in the Royal Air Force in 1948. Since 1955 he has been a member of the faculty at the California Institute of Technology, Pasadena, Calif., where he is now Professor of Electrical Engineering. In 1965-1966 he spent a year lecturing and consulting at some two dozen universities and companies in seven European countries. $\mathrm{He}$ is well known as author, lecturer, and consultant in solid-state devices and circuits. His current research interests are in electronic power processing systems. His publications include a book on solid-state device theory and another on differential amplifiers.

Dr. Middlebrook is a member of Sigma Xi, and is Chairman of the Board of Electric Motion Control Corporation International. 